\title{
Flexible Printed Organic Electrochemical Transistors for the Detection of Uric Acid in Artificial Wound Exudate
}

Marina Galliani, Chiara Diacci, Marcello Berto, Matteo Sensi, Valerio Beni, Magnus Berggren, Marco Borsari, Daniel Simon, Fabio Biscarini and Carlo A. Bortolotti

The self-archived postprint version of this journal article is available at Linköping University Institutional Repository (DiVA):

http://urn.kb.se/resolve?urn=urn:nbn:se:liu:diva-171033

N.B.: When citing this work, cite the original publication.

Galliani, M., Diacci, C., Berto, M., Sensi, M., Beni, V., Berggren, M., Borsari, M., Simon, D., Biscarini, F., Bortolotti, C. A., (2020), Flexible Printed Organic Electrochemical Transistors for the Detection of Uric Acid in Artificial Wound Exudate, Advanced Materials Interfaces, , 2001218.

https://doi.org/10.1002/admi.202001218

Original publication available at:

https://doi.org/10.1002/admi.202001218

Copyright: Wiley

https://www.wiley.com/en-gb 


\title{
Flexible Printed Organic Electrochemical Transistors for the Detection of Uric Acid in Artificial Wound Exudate
}

\author{
Marina Galliani, Chiara Diacci, Marcello Berto, Matteo Sensi, Valerio Beni, Magnus \\ Berggren, Marco Borsari, Daniel T. Simon, Fabio Biscarini, Carlo A. Bortolotti* .
}

Marina Galliani, Chiara Diacci, Dr. Marcello Berto, Dr. Matteo Sensi, Prof. Fabio Biscarini and Dr. Carlo A. Bortolotti.

Dipartimento di Scienze della Vita, Università di Modena e Reggio Emilia, Via Campi 103, 41125 Modena, Italy.

E-mail: carloaugusto.bortolotti@unimore.it

Chiara Diacci, Prof. Magnus Berggren and Prof. Daniel T. Simon.

Laboratory of Organic Electronics, Department of Science and Technology, Linköping University, SE-601 74 Norrköping, Sweden.

Prof. Fabio Biscarini.

Istituto Italiano di Tecnologia - Center for Translational Neurophysiology, Via Fossato di Mortara 17-19, 44121 Ferrara, Italy.

Prof. Marco Borsari.

Dipartimento di Scienze Chimiche e Geologiche, Università di Modena e Reggio Emilia, Via Campi 103, 41125 Modena, Italy.

Dr. Valerio Beni.

Department of Smart Hardware, RISE AB, Research Institutes of Sweden, Norrköping, Sweden.

Keywords: Organic Electrochemical Transistor, enzymatic detection, uric acid, gelatin, wound care.

Low-cost, minimally invasive sensors able to provide real time monitoring of wound infection can enable the optimization of healthcare resources in chronic wounds management. Printed organic electrochemical transistors (OECTs) are valuable candidates for this application. Here, a novel biosensor, based on OECT, for monitoring uric acid (UA), which is a bacterial infection biomarker in wounds, is demonstrated in artificial wound exudate. The sensor exploits the 


\section{WILEY-VCH}

enzymatic conversion of UA to 5-hydroxyisourate, catalysed by Uricase entrapped in a dualionic-layer hydrogel membrane casted onto the gate. The sensor response is based on the catalytic oxidation of the hydrogen peroxide, generated as part of the Uricase regeneration process, at the Pt modified gate. The proposed dual membrane avoids the occurrence of nonspecific faradic reactions as, for example the direct oxidation of UA or others electroactive molecules that would introduce a potentially false negative response. The biosensor is robust and its response is reproducible both in PBS buffer and in complex solutions mimicking the wound exudate. The sensor has a high sensitivity in the range encompassing the pathological levels of UA in wounds $(<200 \mu \mathrm{M})$ exhibits a limit of detection of $4.5 \mu \mathrm{M}$ in artificial wound exudate. All these characteristics make this OECT-based biosensor attractive for wound monitoring interfaced to the patient.

\section{Introduction}

Wound care is an important medical treatment: if neglected, it may slow down or hamper the healing process ${ }^{[1,2]}$ and possibly lead to serious complications ${ }^{[3]}$. Advanced wound care technologies envision the acquisition and communication of data about the wound status directly from the patient home through smart bandages. A slower wound healing process can arise from a variety of endogenous or exogenous factors, including bacterial infections. During the healing process of a wound, exogenous bacteria and/or the ones who normally belong to the skin microbiota (e.g. S. aureus., E. coli, P. aeruginosa) can reach the underlying tissues and proliferate, causing a prolonged inflammation which, in the worst case, leads to a chronic status of the wound ${ }^{[4]}$. Smart bandages have been developed to monitor generic physiological indicators such as $\mathrm{pH}^{[5,6]}$, temperature ${ }^{[7,8]}$, oxygen ${ }^{[9]}$ or moisture level ${ }^{[10]}$. Other indicators of wound infections and healing processes are variations of the level of metalloproteinases ${ }^{[11]}$, cytokines ${ }^{[12]}$ and uric acid (UA) ${ }^{[13]}$. The latter, in the form of urate, is present in wound exudate in concentrations varying between $220 \mu \mathrm{M}$ and $750 \mu \mathrm{M}^{[14]}$. The human body cannot catabolise 


\section{WILEY-VCH}

UA since it lacks a specific enzyme and UA is physiologically eliminated through urine. As a consequence of bad nutrition and metabolic disorders ${ }^{[15,16]}$ or diseases like cancer ${ }^{[17]}$ or diabetes ${ }^{[18]}$, UA levels in bodily fluids can increase leading to gout ${ }^{[19,20]}$ and urate crystals precipitation in joints, kidney and other tissues ${ }^{[19]}$. On the contrary, some bacteria, such as Pseudomonas aeruginosa, can specifically metabolise UA ${ }^{[13,21,22]}$ with subsequent decrease of its concentration in the wound exudate below $200 \mu \mathrm{M}$. As a result, the UA level in skin wound can be monitored as an effective marker of bacterial infection.

Label-free biosensors based on organic (semi-)conductive polymers may represent ideal devices to be interfaced with wound environment: they detect complex biological signals in aqueous media and translate them into an electronic output; they are operated at low voltages and require low power sources and they can be fabricated into flexible materials ${ }^{[23-26]}$ with large-area techniques such as screen printing ${ }^{[27-30]}$ and inkjet printing ${ }^{[31]}$ on low-cost substrates (paper, plastic foils, textiles). Moreover, they can be manufactured as impalpable patches adhering on the skin to record physiological signals ${ }^{[32-34]}$

Biosensors based on organic electrochemical transistors (OECTs) biosensors are particularly appealing for those applications that, while not requiring ultra-low limits of detection, benefit from other features offered by this architecture, such as easy integration with readout circuits, stability, amplification, and the possibility of controlling the dynamic range through device design. OECTs were already demonstrated for detection of metabolites ${ }^{[35-38]}$ in complex media, cytokines ${ }^{[39]}$ and neurotransmitters ${ }^{[40,41]}$.

The present work demonstrates a low-cost and disposable printed OECT sensor for UA detection in complex solutions mimicking wound exudate. The OECT-based sensors are screen printed on flexible PET (polyethylene terephthalate) foil ${ }^{[42]}$, featuring poly(3,4-ethylenedioxythiophene):poly(styrenesulfonate) PEDOT:PSS as the conducting polymer for the channel and amorphous carbon as the gate electrode. Amorphous carbon is a suitable material 


\section{WILEY-VCH}

for the gate electrode since it is cost effective, its printing process is well established and it can be employed as a substrate for further modification via electrochemical deposition of metals, such as platinum ${ }^{[43]}$, or gold ${ }^{[44]}$. Our approach for UA detection is based on the catalytic activity of the enzyme Urate oxidase (UOx, also known as Uricase) that ultimately produces hydrogen peroxide. One of the innovative features of our device architecture with respect to previously reported detection of UA with OECT ${ }^{[23]}$ is the architecture of the sensing element, composed by two layers of hydrogels integrated on top of the gate electrode surface. The two hydrogel layers consist of a polycation and a polyanion network, one on top of the other. Their function is to create a charge-selective barrier that prevents the flow of charged molecules to the gate electrode, suppressing in this way parasitic Faradic reactions due to the oxidation of electroactive molecules present in the measuring medium. Such functionalization is aimed to allow only hydrogen peroxide to diffuse to the gate, where it can be oxidized to oxygen, thus ensuring the specificity of the sensor response. The dynamic range observed spans both the pathological and physiological uric acid levels in wounds and exhibits robust response also in complex media of medical relevance. This device architecture, which is simple to manufacture and to scale up into high throughput production, may represent a disposable and easy to use solution to be integrated in adhesive strips or bandages for advanced wound care systems.

\section{Results and Discussion}

The device layout exhibits a planar geometry with all-printed amorphous carbon gate, source and drain electrodes, the latter bridged by a printed channel made of PEDOT:PSS conducting polymer (Figure 1A). In order to enhance gate modulation capability and catalyse the oxidation of hydrogen peroxide within the $0-1 \mathrm{~V}$ potential range, a Pt layer was electrodeposited on the gate $^{[45]}$ (Fig. S1A, Supporting Information). In $50 \mathrm{mM}$ Phosphate Buffer Saline (PBS), the

carbon gate exhibits no oxidation processes below $1 \mathrm{~V}^{[44]}$, also in the presence of the Pt layer (Fig. S1B). Both the transfer characteristics ( $\mathrm{I}_{\mathrm{DS}} \mathrm{vs} . \mathrm{V}_{\mathrm{GS}}$ at constant $\mathrm{V}_{\mathrm{DS}}$ ) and the output curves 


\section{WILEY-VCH}

(IDS vs. $\mathrm{V}_{\mathrm{DS}}$ at constant $\mathrm{V}_{\mathrm{GS}}$ ) show that, at variance with what can be achieved with the $\mathrm{C}$ gate, the $\mathrm{C} / \mathrm{Pt}$ gate can lead to drain current modulation as expected from a depletion-mode OECT. The gate electrode was further modified to enable enzyme immobilization and enhance sensor selectivity: a negatively charged gelatin B layer is deposited onto the platinized gate electrode and subsequently a positively charged gelatin A layer, with cross-linked Uricase, is deposited on the gelatin B membrane (Figure 1B). Gelatin A and B are natural hydrogels derived from porcine skin; they are biocompatible and provide a robust environment for enzymes ${ }^{[30,46-49]}$. The use of gelatin represents a valuable functionalization strategy for wound-monitoring biosensors, as they were already used for wound patches and scaffolds ${ }^{[50-52]}$. The gelatin A layer is permeable to UA and therefore allows it to reach Uricase, which catalyses its oxidation to 5-hydroxyisourate that finally spontaneously converts into allantoin (Fig. 2a) ${ }^{[53]}$. The gelatin A layer, formed by a positively charged polymeric network with mobile anions to balance its charge, also enhances selectivity by hindering diffusion of cations to the gate.

A
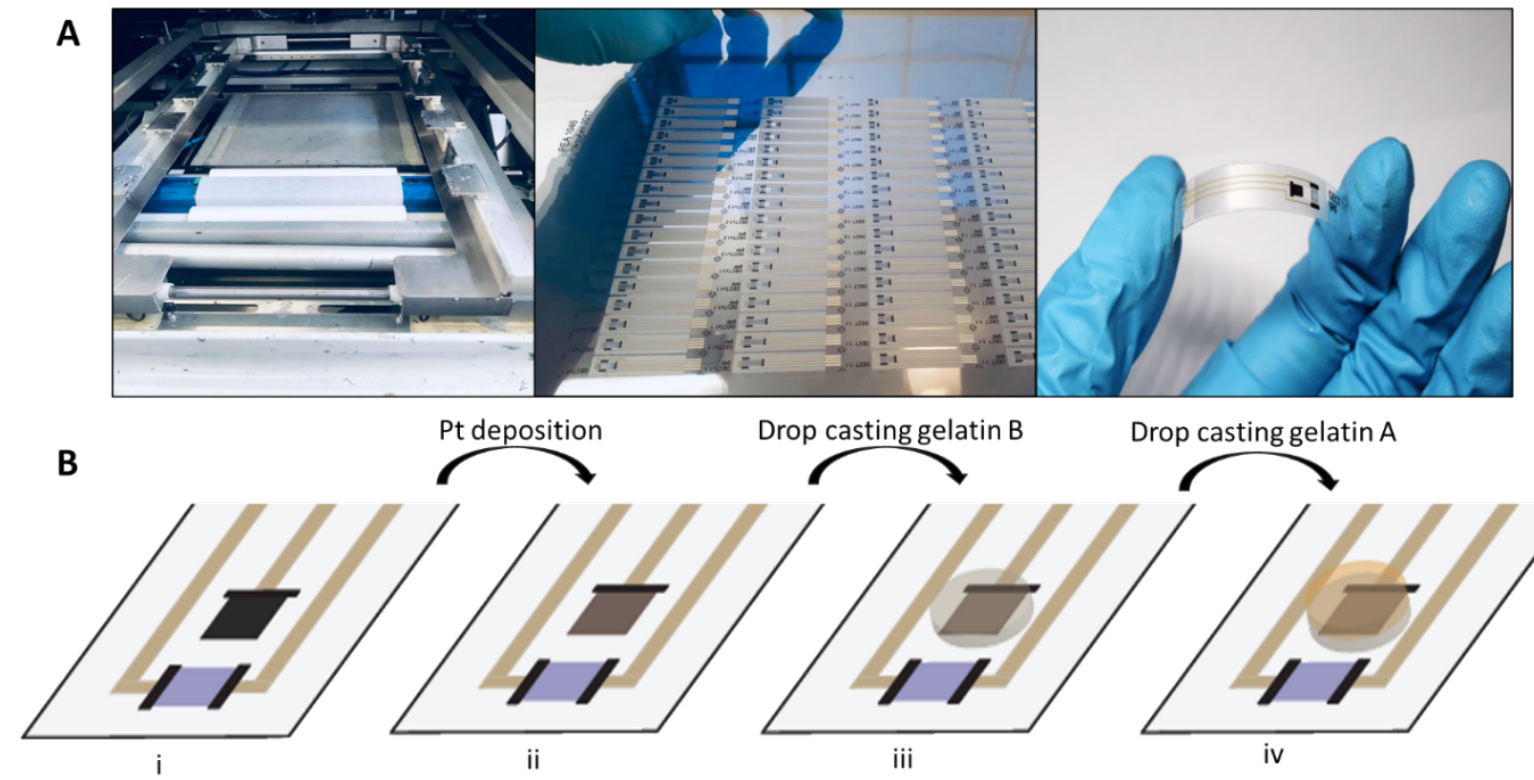

Drop casting gelatin $\mathrm{A}$

Drop casting gelatin $B$
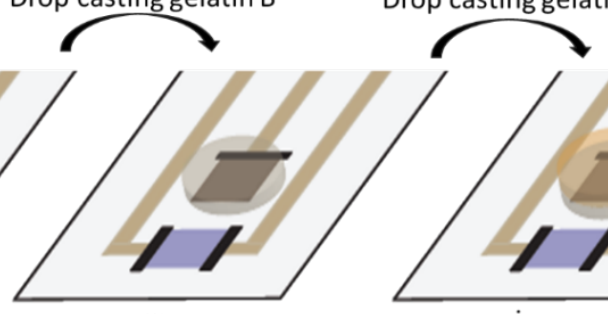

iii

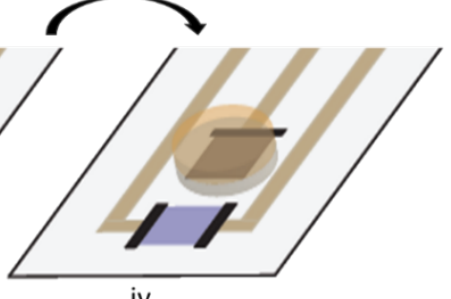

Figure 1: OECT fabrication and functionalization. (A) Screen printing apparatus and printed OECT on PET foil. (B) Steps for the gate functionalization (from left to right): $i \rightarrow$ ii Platinum electrochemical deposition, ii $\rightarrow$ iii drop casting of gelatin B (grey) and iii $\rightarrow$ iv drop casting of gelatin A (orange) cross-linked with Uricase enzymes. 


\section{WILEY-VCH}

The enzyme returns to its resting state through the reduction of molecular oxygen to $\mathrm{H}_{2} \mathrm{O}_{2}$, which is then oxidized at the platinum layer (Figure 2A).

The gelatin B layer, sandwiched between the electrodeposited Pt and gelatin A, is instead formed by a negatively charged network with mobile countercations; as a consequence, its role is to hinder diffusion of anionic electroactive species to the gate, which could provide potential Faradic response.

Our sensing strategy therefore relies on the indirect quantification of UA through oxidation of hydrogen peroxide. Before constructing a dose curve of our device to UA, we first performed a series of control experiments, in the absence of Uricase, to assess the effectiveness of the dualionic-layer hydrogel in allowing $\mathrm{H}_{2} \mathrm{O}_{2}$ diffusion while preventing UA from reaching the gate.

We quantify the sensor response as the signal $\mathrm{S}$, corresponding to the current variation normalized to the current in the absence of analyte, i.e. $\mathrm{S}=\Delta \mathrm{I} / \mathrm{I}=-\left(I_{U A}-I_{0}\right) / I_{0}$, where $\mathrm{I}_{\mathrm{UA}}$ and $\mathrm{I}_{0}$ are the drain current values at a finite molar concentration [UA] and at $[\mathrm{UA}]=0 \mathrm{M}$, respectively. Normalization to the initial current is an effective strategy to minimize device-todevice variations, widely used in organic electronic biosensing ${ }^{[35]}$.

Figure 2B shows the normalized current variation as a function of [UA] for OECT gated by a $\mathrm{C}$ gate with an electrodeposited $\mathrm{Pt}$ layer only $(\mathrm{C} / \mathrm{Pt})$ or further modified by gelatin $\mathrm{B}$ and $\mathrm{A}$ hydrogels $(\mathrm{C} / \mathrm{Pt} / \mathrm{GelB} / \mathrm{GelA})$ with black and blue dots, respectively. It is apparent that UA can be electrochemically oxidised at the $\mathrm{C} / \mathrm{Pt}$ gate, leading to a drain current decrease, while the drain current variation is negligible for $\mathrm{C} / \mathrm{Pt} / \mathrm{GelB} / \mathrm{GelA}$ gated OECT, indicating that direct oxidation of UA at the latter gate is impaired by the two-layer-hydrogel. It is therefore likely that diffusion of other negatively charged species, which could be oxidised in the same potential range at the gate, will be prevented by gelatin $\mathrm{B}$. Figure $2 \mathrm{C}$ depicts the normalized current changes of OECTs gated by $\mathrm{C} / \mathrm{Pt}$ and $\mathrm{C} / \mathrm{Pt} / \mathrm{GelB} / \mathrm{GelA}$ electrodes as a function of $\left[\mathrm{H}_{2} \mathrm{O}_{2}\right]$ : a similar response is obtained in the absence (black circles) and presence (red circles) of the two 


\section{WILEY-VCH}

gelatin layers, suggesting that the dual-ionic-layer hydrogel does not hinder diffusion of hydrogen peroxide to the gate, a necessary requisite for our sensing scheme.

A
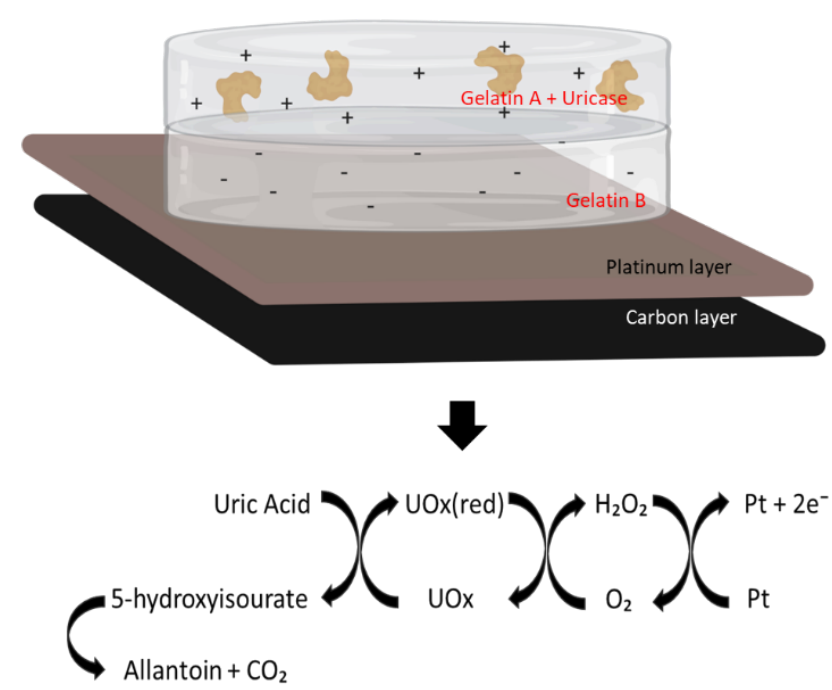
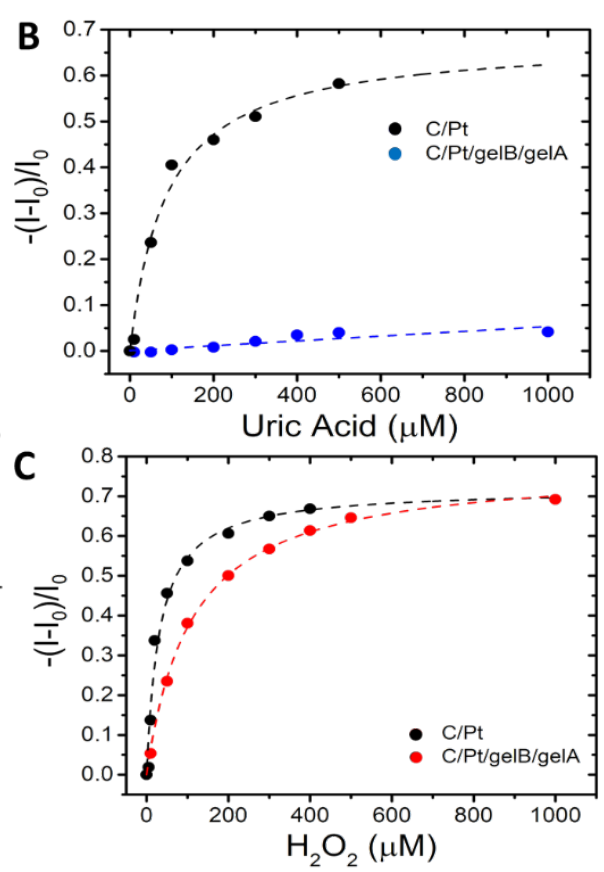

Figure 2: Functionalization strategy. (A) Top: Functionalized gate presenting a platinum layer on top of the carbon printed gate enzyme (the cartoon representation is not on scale). Bottom: the reactions involved in the enzymatic detection of UA. (B) Normalized current variation at fixed $\mathrm{V}_{\mathrm{GS}}=0.5 \mathrm{~V}$, as a function of $[\mathrm{UA}]$ for OECT gated by a bare platinum gate $(\mathrm{C} / \mathrm{Pt}$, black dots) and at a Platinum gate further functionalized gate with Gelatin $\mathrm{A}$ and $\mathrm{B}(\mathrm{C} / \mathrm{Pt} / \mathrm{GelB} / \mathrm{GelA}$, blue dots). (C) Normalized current variation as a function of $\left[\mathrm{H}_{2} \mathrm{O}_{2}\right]$ for OECT gated by $\mathrm{C} / \mathrm{Pt}$ (black dots) and by $\mathrm{C} / \mathrm{Pt} / \mathrm{GelB} / \mathrm{GelA}$ (red dots) electrodes.

We then turned to the detection of UA through quantification of $\mathrm{H}_{2} \mathrm{O}_{2}$ produced in relation to the enzymatic conversion of UA to 5-hydroxyisourate. Since chitosan has been widely reported as a biocompatible matrix to entrap the enzymes ${ }^{[35,54-56]}$, its application, in replacement of gelatin A, in the dual biopolymer hydrogel was tested. Devices featuring the Uricase, entrapped either in gelatin $\mathrm{A}(\mathrm{C} / \mathrm{Pt} / \mathrm{GelB} / \mathrm{GelA}+\mathrm{UOx})$ either in chitosan $(\mathrm{C} / \mathrm{Pt} / \mathrm{GelB} / \mathrm{Chit}+\mathrm{UOx})$ were 


\section{WILEY-VCH}

manufactured and their performances compared. For both OECTs, we measured the real time response to increasing concentration of UA in PBS (Figures $\mathbf{3 A}$ and S2), operating the transistor at constant biases applied at the gate $\left(V_{\mathrm{GS}}=+0.5 \mathrm{~V}\right)$ and at the channel $\left(V_{\mathrm{SD}}=-0.4\right.$ V), since these potential values guarantee high transconductance and therefore the maximum signal amplification ${ }^{[57]}$ (Fig. S2). We noticed that, for each investigated [UA], measurements performed with the Gelatin layers (i.e. for devices gated by $\mathrm{C} / \mathrm{Pt} / \mathrm{GelB} / \mathrm{GelA}+\mathrm{UOx}$ ) reached the steady state response faster when compared to the chitosan functionalization method (Fig. S2). We hypothesized that the slower response from the $\mathrm{C} / \mathrm{Pt} / \mathrm{GelB} / \mathrm{Chit}+\mathrm{UOx}-\mathrm{based}$ OECT might be due to either the extended hydrogen bond network within crosslinked chitosan that might slow down diffusion of $\mathrm{H}_{2} \mathrm{O}_{2}$ towards the gate or to the fact that chitosan at $\mathrm{pH}$ values around neutrality is uncharged, and may therefore collapse to an insoluble, denser layer ${ }^{[58]}$.

Therefore, we relied on the functionalization strategy based on the gelatin A/gelatin B hydrogels to perform multiple experiments to increase the data statistics. For each IDS vs time measurement (Figure 3A), we extracted the sensor response S, expressed as the normalized drain current change, as previously described, for UA concentrations ranging from $10 \mu \mathrm{M}$ to 1 $\mathrm{mM}$, and we averaged the results obtained with four independent measurements to construct the dose curve in Figure 3B.

The response increases monotonically in the investigated concentration range and tends to saturate for [UA] approaching the millimolar range; the estimated Limit of Detection (LOD) is $10.5 \mu \mathrm{M}$. Most notably, the sensor can quantify UA levels both in the physiological (green box) and in pathological range (red box), the latter associated with lower [UA] values typically found in wounds infected by bacteria ${ }^{[13]}$.

The working principle of electrolyte gated organic transistors, and in particular of OECT based biosensor, is yet to be fully elucidated. Our interpretation of the device response to $\mathrm{H}_{2} \mathrm{O}_{2}$ (either produced by Uricase or directly added to the electrolyte solution as in the aforementioned control experiments) can be depicted as follows. During non-Faradic OECT operational regime, 


\section{WILEY-VCH}

a positive bias is applied to the gate electrode, forcing cations, present in the electrolyte, to drift into the porous PEDOT:PSS channel, thus lowering the channel conductivity (dedoping). Conversely, here, in the presence of UA and Uricase, the OECT works in the Faradic regime: $\mathrm{H}_{2} \mathrm{O}_{2}$ oxidation takes place at the gate and might lead to concomitant reduction of PEDOT, with accompanying penetration of cations in the semiconductor channel, resulting in a decrease of the drain current ${ }^{[40,44]}$. Alternatively, the working principle underlying the operation of OECTs as enzyme-based biosensors has been previously described ${ }^{[59]}$ in terms of increased electrolyte potential caused by an increase in $\mathrm{H}_{2} \mathrm{O}_{2}$ concentration through the Nernst equation, resulting in a more positive effective gate potential, which impacts on the cation drift in the porous PEDOT:PSS channel and thus decreases the drain current ${ }^{[60-62]}$.

To mimic the complex environment of an infected wound, we performed measurements in an aqueous solution serving as artificial wound exudate ${ }^{[14]}$, the composition of which can be found in the Materials and Methods section. We first assessed whether the $\mathrm{C} / \mathrm{Pt} / \mathrm{GelB} / \mathrm{GelA}+\mathrm{UOx}-$ gated OECTs could be operated even in such a more complex medium: as witnessed by the transfer and output characteristics in Fig. S3, current modulation in the same voltage range can still be achieved, and this result indicates that our OECTs are robust enough to withstand operation also in complex media, such as an artificial biological fluid. Nevertheless, differences in the electrical performances of the device when switching from operation in PBS to artificial exudate can be observed this finding might indicate that the device is somehow sensitive to the nature of the matrix, likely due to changes in the double layer capacitance as the ionic composition of the medium is altered.

We then performed multiple real time measurements in artificial wound exudate solutions spiked with UA in the same [UA] range that was previously explored in PBS. A typical IDs vs. time response to increasing [UA] values in artificial wound exudate, provided in Figure S4, indicates that the biosensor can be used to detect increasing uric acid concentration, even in an environment mimicking a biological milieu. The dose curve in the artificial wound exudate is 


\section{WILEY-VCH}

provided in Figure 3B: the biosensor response closely parallels the one obtained in PBS and the calculated LOD is as low as $4.5 \mu \mathrm{M}$ in the artificial wound exudate. The derivative of the dosecurve response shows that our sensor exhibits the highest sensitivity below $200 \mu \mathrm{M}$, viz. in the pathological region (red box), and lower sensitivity in the physiological range (200 $\mu \mathrm{M}-700$ $\mu \mathrm{M}$, green box).


Figure 3: Uric acid detection in complex media. (A) Real time drain current changes at increasing uric acid concentrations in PBS buffer. (B) Normalized current change (signal $\mathrm{S}=-$ $\left.\left.\left(I_{U A}-I_{0}\right) / I_{0},\right)\right)$ in PBS (black dots) and in artificial wound exudate (violet dots) as a function of [UA]. Error bars represent the standard error from four measurements. The inset shows the derivative of the signal S in PBS and in artificial wound exudate as black and violet curves, respectively. Pathological and physiological levels are marked as red and green boxes, respectively.

\section{Conclusion}

We reported a printed UA biosensor based on OECT architecture, which exploits a biocompatible dual layer functionalization strategy to both immobilize the selective biorecognition element and to hinder parasitic reactions from charged interfering compounds. We demonstrated that our OECT-based biosensor can operate in artificial biological fluids, still 


\section{WILEY-VCH}

maintaining similar sensitivity as for model solutions like PBS buffer. In addition, the OECT biosensor based on porcine-skin derived gelatin shows a faster response when compared to other substrates often used for enzyme entrapping. The device architecture and performance make the demonstrated device particularly attractive for wound infection monitoring. In view of future system integrations, these biosensors can be further engineered and coupled with devices for the local delivery of antibiotics, thus realizing a closed loop system loco-regional approach for wound care.

Moreover, the different electrical response upon switching from PBS to the artificial wound exudate might open to the possibility to monitor changes in the levels of polyelectrolytes in complex samples besides monitoring the specific response enabled by the presence of the biorecognition unit, possibly disentangling the response of redox active species (Faradic) from that of other compounds (non-Faradic) through the use of multivariate analysis, although this falls beyond the scope of the present paper. Eventually, the gate modification by a bipolar membrane (a cation- and an anion-selective membrane in contact with one another, as described in the present work) could be further exploited by getting inspiration from the ion bipolar membrane diodes architecture ${ }^{[63]}$, which is also based on a stack of a cation- and anionexchange membranes and that was successfully demonstrated as logic element and integrated with electrochromic display ${ }^{[64]}$.

\section{Experimental Section}

\subsection{Device fabrication}

OECTs were manufactured by screen-printing technique. As substrate was used a $125 \mu \mathrm{m}$ thick PET (polyethylene terephthalate) foil, thermally treated (45mins at $140{ }^{\circ} \mathrm{C}$ ) to confer more resistance to deformation by subsequent curing steps. Conductive tracks were printed using an Ag ink (Ag5000, DuPont, UK), whereas carbon contact pads and the gate were printed using a 


\section{WILEY-VCH}

commercial carbon ink (C2130307DI, Gwent, UK). The organic semiconductor was printed in the channel using a commercial PEDOT: PSS ink (CleviosTM SV3, Heraeus Group, Germany). As the final step, the printed OECTs were coated with a dielectric layer (5018, DuPont, UK) leaving uncovered the gate and channel areas and the connecting pads. Devices were printed using a semi-automatic screen printer (DEK Horizon 03i printer, ASM Assembly Systems $\mathrm{GmbH}$, Germany). Both the channel and the gate exposed areas measure $4 \mathrm{~mm}^{2}$.

\subsection{Device functionalization}

A potassium hexachloroplatinate $\left(\mathrm{K}_{2} \mathrm{PtCl}_{6}\right.$, Sigma Aldrich) $10 \mathrm{mM}$ solution in $\mathrm{H}_{2} \mathrm{SO}_{4} 0.5 \mathrm{M}$ was used, as platinum source, to modify the OECT carbon electrode. The electrodeposition was performed at a fixed voltage of $-0.5 \mathrm{~V}$ with a potentiostat $(\mathrm{CH}$ Instrument Potentiostat $760 \mathrm{c}$ model), using a micro $\mathrm{Ag} / \mathrm{AgCl}$ reference electrode (Elbatech, Livorno Italy) and a platinum wire as the counter electrode. The negatively charged layer was made of Gelatin from porcine skin (gel strength 300, type-B, Sigma Aldrich). A 5\% Gelatin type B aqueous solution was prepared from dissolving dried gelatin in $50 \mathrm{mM} \mathrm{PBS}$ at $60^{\circ} \mathrm{C}$ for $1 \mathrm{~h}$. A $3 \mu 1$ drop of the former solution was cast on the gate area and a $1.2 \mu 1$ drop of $2.5 \%$ glutaraldehyde water solution, used as crosslinking agent. The device was then stored at $4^{\circ} \mathrm{C}$ for 24 hours. A second layer of gelatin was then drop casted on the device. Uricase enzyme from Bacillus fastidiosus is immobilized in a gelatin type-A (Sigma Aldrich), positively charged at physiologic $\mathrm{pH}$, with glutaraldehyde as the crosslinking agent. A mixture of $3 \%$ gelatin type A PBS solution $(20 \mu 1), 5 \mathrm{mg} / \mathrm{ml}$ enzyme TRIS buffer solution $(20 \mu \mathrm{l})$ and $2.5 \%$ glutaraldehyde aqueous solution $(16 \mu \mathrm{l})$ was prepared; then a $6 \mu 1$ drop the mixed solution was casted on the gate area. The devices were left 4 hours at $4^{\circ} \mathrm{C}$ before use.

4.3 Electrical characterization and data analysis 


\section{WILEY-VCH}

Electric measurements were performed using an Agilent B2912A Source Measure Unit in phosphate buffer saline (PBS) $50 \mathrm{mM}, \mathrm{pH} 7.4$, at room temperature. Transfer characteristics were recorded sweeping the gate voltage $\left(\mathrm{V}_{\mathrm{GS}}\right)$ from $0 \mathrm{~V}$ to $1 \mathrm{~V}$ and fixed drain-source voltage $\left(\mathrm{V}_{\mathrm{DS}}\right)$ of $-0.4 \mathrm{~V}$. Output characteristics were measured scanning the $\mathrm{V}_{\mathrm{DS}}$ from $0 \mathrm{~V}$ to $-0.8 \mathrm{~V}$, while sweeping the $\mathrm{V}_{\mathrm{GS}}$ from $0 \mathrm{~V}$ to $1 \mathrm{~V}$ with 10 steps of $0.1 \mathrm{~V}$ each. The response of each sensor to the addition of the analyte, was measured in real-time at constant bias mode: $\mathrm{V}_{\mathrm{GS}}$ and $\mathrm{V}_{\mathrm{DS}}$ were fixed at $+0.5 \mathrm{~V}$ and $-0.4 \mathrm{~V}$, respectively, and the $\mathrm{I}_{\mathrm{DS}}$ was registered as a function of time.

To obtain the normalized response $\mathrm{S}$ to increasing UA concentrations as $S=\Delta I / I=-\left(I_{U A}-I_{0}\right) / I_{0}$, $I_{U A}$ was taken as the drain current recorded 300 seconds after each UA injection, while $I_{0}$ is the current recorded in the absence of analyte. $1 \mathrm{mM}$ concentration in PBS normalized response was obtained with the average of only two devices.

The limit of detection (LOD) is the lowest concentration detectable from the device and has been calculated as LOD $=\mathrm{S}_{\text {buffer }}+3 \sigma$, where $\mathrm{S}_{\text {buffer }}$ is the signal for PBS and artificial wound exudate in absence of analyte and $\sigma$ is their standard deviation. $\sigma$ has been obtained from the PBS and artificial wound exudate IDS current values during the stabilization time.

\subsection{Artificial wound exudate}

The artificial wound exudate medium was prepared by dissolving in water sodium chloride 124 $\mathrm{mM}$, magnesium chloride $831 \mu \mathrm{M}$, calcium chloride $2.48 \mathrm{mM}$, sodium bicarbonate $36.8 \mathrm{mM}$, glucose $5 \mathrm{mM}$, albumin $150 \mu \mathrm{M}$, lactic acid 90\% $10 \mu \mathrm{M}$. Measured pH: 6.72 (ref 14).

\section{Supporting Information}

Supporting Information is available from the Wiley Online Library or from the author.

\section{Acknowledgements}




\section{WILEY-VCH}

VB would like to unknowledge the Swedish Foundation for Strategic Research (BioCom Lab, grant RIT15-0119) for financial support. DS, MB, CD: Support for this research was provided by the Swedish Foundation for Strategic Research and the Knut and Alice Wallenberg Foundation. This work was conducted within the framework of the BioCom Lab projects.

Additional assistance with mask design and printing was provided by Ulrika Linderhed and Marie Nilsson at RISE AB, Norrköping, Sweden.

Received: ((will be filled in by the editorial staff))

Revised: ((will be filled in by the editorial staff)) Published online: ((will be filled in by the editorial staff))

\section{References}

[1] C. E. Fife, M. J. Carter, D. Walker, Wound Repair Regen. 2010, 18, 154.

[2] C. K. Sen, G. M. Gordillo, S. Roy, R. Kirsner, L. Lambert, T. K. Hunt, F. Gottrup, G.

C. Gurtner, M. T. Longaker, Wound Repair Regen. 2009, 17, 763.

[3] H. Derakhshandeh, S. S. Kashaf, F. Aghabaglou, I. O. Ghanavati, A. Tamayol, Trends Biotechnol. 2018, 36, 1259.

[4] I. Negut, V. Grumezescu, A. M. Grumezescu, Molecules 2018, 23, 1.

[5] T. Guinovart, G. Valdés-Ramírez, J. R. Windmiller, F. J. Andrade, J. Wang, Electroanalysis 2014, 26, 1345.

[6] P. Salvo, V. Dini, A. Kirchhain, A. Janowska, T. Oranges, A. Chiricozzi, T. Lomonaco, F. Di Francesco, M. Romanelli, Sensors (Switzerland) 2017, 17, 10.

[7] P. Salvo, N. Calisi, B. Melai, B. Cortigiani, M. Mannini, A. Caneschi, G. Lorenzetti, C. Paoletti, T. Lomonaco, A. Paolicchi, I. Scataglini, V. Dini, M. Romanelli, R. Fuoco, F. Di Francesco, Biosens. Bioelectron. 2017, 91, 870.

[8] Y. Moser, M. A. M. Gijs, TRANSDUCERS EUROSENSORS '07 - 4th Int. Conf. SolidState Sensors, Actuators Microsystems 2007, 16, 2279. 


\section{WILEY-VCH}

[9] S. Schreml, R. J. Meier, M. Kirschbaum, S. C. Kong, S. Gehmert, O. Felthaus, S. Küchler, J. R. Sharpe, K. Wöltje, K. T. Weiß, M. Albert, U. Seidl, J. Schröder, C. Morsczeck, L. Prantl, C. Duschl, S. F. Pedersen, M. Gosau, M. Berneburg, O. S. Wolfbeis, M. Landthaler, P. Babilas, Theranostics 2014, 4, 721.

[10] D. McColl, B. Cartlidge, P. Connolly, Int. J. Surg. 2007, 5, 316.

[11] M. P. Caley, V. L. C. Martins, E. A. O’Toole, Adv. Wound Care 2015, 4, 225.

[12] S. Barrientos, O. Stojadinovic, M. S. Golinko, H. Brem, M. Tomic-Canic, Wound Repair Regen. 2008, 16, 585.

[13] P. Kassal, J. Kim, R. Kumar, W. R. De Araujo, I. M. Steinberg, M. D. Steinberg, J. Wang, Electrochem. commun. 2015, 56, 6.

[14] N. J. Trengove, S. R. Langton, M. C. Stacey, Wound Repair Regen. 1996, 4, 234.

[15] P. Cirillo, W. Sato, S. Reungjui, M. Heinig, M. Gersch, Y. Sautin, T. Nakagawa, R. J. Johnson, J. Am. Soc. Nephrol. 2006, 17, S165 LP.

[16] H. K. Choi, K. Atkinson, E. W. Karlson, W. Willett, G. Curhan, N. Engl. J. Med. 2004, $350,1093$.

[17] S. C. Howard, D. P. Jones, C.-H. Pui, N. Engl. J. Med. 2011, 364, 1844.

[18] F. P. Cappuccio, P. Strazzullo, E. Farinaro, M. Trevisan, JAMA 1993, 270, 354.

[19] P. Richette, T. Bardin, Lancet 2010, 375, 318.

[20] M. Heinig, R. J. Johnson, Cleve. Clin. J. Med. 2006, 73, 1059.

[21] T. R. Dargaville, B. L. Farrugia, J. A. Broadbent, S. Pace, Z. Upton, N. H. Voelcker, Biosens. Bioelectron. 2013, 41, 30.

[22] M. A. Rouf, R. F. Lomprey, 1968, 96, 617.

[23] C. Liao, C. Mak, M. Zhang, H. L. W. Chan, F. Yan, Adv. Mater. 2015, 27, 676.

[24] L. J. Currano, F. C. Sage, M. Hagedon, L. Hamilton, J. Patrone, K. Gerasopoulos, Sci. Rep. 2018, 8, 15890.

[25] D. Khodagholy, V. F. Curto, K. J. Fraser, M. Gurfinkel, R. Byrne, D. Diamond, G. G. 


\section{WILEY-VCH}

Malliaras, F. Benito-Lopez, R. M. Owens, J. Mater. Chem. 2012, 22, 4440.

[26] C. Yao, Q. Li, J. Guo, F. Yan, I. M. Hsing, Adv. Healthc. Mater. 2015, 4, 528.

[27] L. Contat-Rodrigo, C. Pérez-Fuster, J. V. Lidón-Roger, A. Bonfiglio, E. García-Breijo, Org. Electron. physics, Mater. Appl. 2017, 45, 89.

[28] A. Aliane, G. Marchand, V. F. Curto, R. Coppard, R. M. Owens, X. Strakosas, G. G. Malliaras, P. Mailley, G. Scheiblin, MRS Commun. 2015, 5, 507.

[29] G. Scheiblin, A. Aliane, R. Coppard, R. M. Owens, P. Mailley, G. G. Malliaras, SPIE Org. Photonics + Electron. 2015, 9568, 95681E.

[30] M. Berto, C. Diacci, L. Theuer, M. Di Lauro, D. T. Simon, M. Berggren, F. Biscarini, V. Beni, C. A. Bortolotti, Flex. Print. Electron. 2018, 3, DOI 10.1088/2058$8585 / \mathrm{aac} 8 \mathrm{a} 8$.

[31] G. Mattana, A. Loi, M. Woytasik, M. Barbaro, V. Noël, B. Piro, Adv. Mater. Technol. 2017, 2,1 .

[32] O. J. Rauhala, S. Dominguez, G. D. Spyropoulos, J. J. Ferrero, T. R. Boyers, P. Jastrzebska-perfect, C. Cea, D. Khodagholy, J. N. Gelinas, 2019, 1900663, DOI 10.1002/admt.201900663.

[33] C. Cea, G. D. Spyropoulos, P. Jastrzebska-Perfect, J. J. Ferrero, J. N. Gelinas, D. Khodagholy, Nat. Mater. 2020, 1.

[34] A. Campana, T. Cramer, D. T. Simon, M. Berggren, F. Biscarini, Adv. Mater. 2014, 26, 3874.

[35] C. Diacci, J. W. Lee, P. Janson, G. Dufil, G. Méhes, M. Berggren, D. T. Simon, E. Stavrinidou, Adv. Mater. Technol. 2020, 5, DOI 10.1002/admt.201900262.

[36] A. M. Pappa, V. F. Curto, M. Braendlein, X. Strakosas, M. J. Donahue, M. Fiocchi, G. G. Malliaras, R. M. Owens, Adv. Healthc. Mater. 2016, 5, 2295.

[37] X. Strakosas, M. Huerta, M. J. Donahue, A. Hama, A. M. Pappa, M. Ferro, M. Ramuz, J. Rivnay, R. M. Owens, J. Appl. Polym. Sci. 2017, 134, 1. 


\section{WILEY-VCH}

[38] Y. Ding, L. Zheng, G. Zhang, L. Qiu, G. Wang, L. Zhang, D. Wu, C. Xiong, H. Lu, Biosens. Bioelectron. 2017, 100, 235.

[39] D. Gentili, P. D’Angelo, F. Militano, R. Mazzei, T. Poerio, M. Brucale, G. Tarabella, S. Bonetti, S. L. Marasso, M. Cocuzza, L. Giorno, S. Iannotta, M. Cavallini, J. Mater. Chem. B 2018, 6, 5400.

[40] L. Kergoat, B. Piro, D. T. Simon, M. C. Pham, V. Noël, M. Berggren, Adv. Mater. 2014, 26, 5658 .

[41] H. Tang, P. Lin, H. L. W. Chan, F. Yan, Biosens. Bioelectron. 2011, 26, 4559.

[42] Z. Bao, Y. Feng, A. Dodabalapur, V. R. Raju, A. J. Lovinger, Chem. Mater. 1997, 9, 1299.

[43] L. Theuer, J. Randek, S. Junne, P. Neubauer, C. F. Mandenius, V. Beni, Processes 2020, 8, DOI 10.3390/pr8030321.

[44] M. Sensi, M. Berto, A. Candini, A. Liscio, A. Cossarizza, V. Beni, F. Biscarini, C. A. Bortolotti, ACS Omega 2019, 4, 5374.

[45] K. J. J. Mayrhofer, J. C. Meier, W. B. Schneider, U. Benedikt, A. A. Auer, P. U. Biedermann, I. Katsounaros, Phys. Chem. Chem. Phys. 2012, 14, 7384.

[46] A. P. Periasamy, Y. J. Chang, S. M. Chen, Bioelectrochemistry 2011, 80, 114.

[47] S. Karacaoğlu, S. Timur, A. Telefoncu, Artif. Cells. Blood Substit. Immobil. Biotechnol. 2003, 31, 357.

[48] P. Jolly, A. Miodek, D. Yang, L. Chen, M. D. Lloyd, P. Estrela, ACS Sensors 2016, acssensors.6b00443.

[49] D. Bagal-Kestwal, R. M. Kestwal, B. H. Chiang, M. S. Karve, Sensors Actuators, B Chem. 2011, 160, 1026.

[50] E. Vatankhah, M. P. Prabhakaran, G. Jin, L. G. Mobarakeh, S. Ramakrishna, J. Biomater. Appl. 2014, 28, 909.

[51] K. Ulubayram, A. N. Cakar, P. Korkusuz, C. Ertan, N. Hasirci, Biomaterials 2001, 22, 


\section{WILEY-VCH}

1345.

[52] Y. S. Choi, S. B. Lee, S. R. Hong, Y. M. Lee, K. W. Song, M. H. Park, J. Mater. Sci. Mater. Med. 2001, 12, 67.

[53] P. E. Erden, E. Kiliç, Talanta 2013, 107, 312.

[54] C. Liao, M. Zhang, L. Niu, Z. Zheng, F. Yan, J. Mater. Chem. B 2014, 2, 191.

[55] S. Islam, M. A. R. Bhuiyan, M. N. Islam, J. Polym. Environ. 2017, $25,854$.

[56] J. R. Anusha, C. J. Raj, B. B. Cho, A. T. Fleming, K. H. Yu, B. C. Kim, Sensors Actuators, B Chem. 2015, 215, 536.

[57] D. A. Bernards, G. G. Malliaras, Adv. Funct. Mater. 2007, 17, 3538.

[58] H.-S. Lee, M. Q. Yee, Y. Y. Eckmann, N. J. Hickok, D. M. Eckmann, R. J. Composto, J. Mater. Chem. 2012, 22, 19605.

[59] D. A. Bernards, D. J. Macaya, M. Nikolou, J. A. DeFranco, S. Takamatsu, G. G. Malliaras, J. Mater. Chem. 2008, 18, 116.

[60] O. Yaghmazadeh, F. Cicoira, D. A. Bernards, S. Y. Yang, Y. Bonnassieux, G. G. Malliaras, J. Polym. Sci. Part B Polym. Phys. 2011, 49, 34.

[61] G. G. Malliaras, C. Bernard, C. K. Ober, T. Doublet, M. E. Welch, J. Polym. Sci. Part A Polym. Chem. 2014, 53, 372.

[62] H. Tang, F. Yan, P. Lin, J. Xu, H. L. W. Chan, Adv. Funct. Mater. 2011, 21, 2264.

[63] E. O. Gabrielsson, K. Tybrandt, M. Berggren, Lab Chip 2012, 12, 2507.

[64] A. Malti, E. O. Gabrielsson, X. Crispin, M. Berggren, Adv. Mater. 2015, 27, 3909.

Copyright WILEY-VCH Verlag GmbH \& Co. KGaA, 69469 Weinheim, Germany, 2018. 


\section{WILEY-VCH}

\section{Supporting informations}

\section{Flexible Printed Organic Electrochemical Transistors for the Detection of Uric Acid in}

\section{Artificial Wound Exudate}

Marina Galliani, Chiara Diacci, Marcello Berto, Matteo Sensi, Valerio Beni, Magnus

Berggren, Marco Borsari, Daniel T. Simon, Fabio Biscarini, Carlo A. Bortolotti*

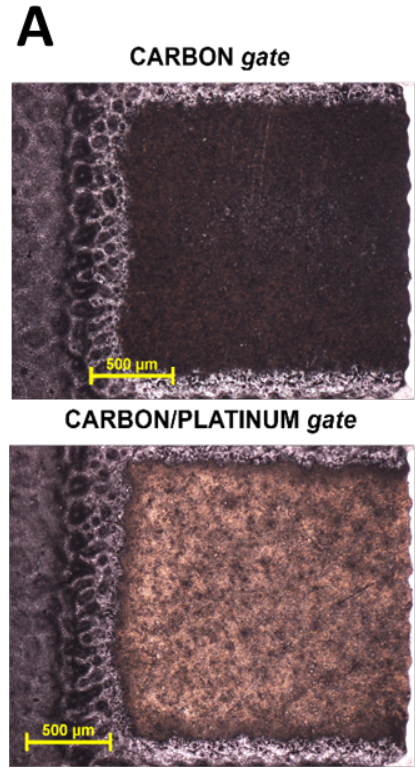

C



B

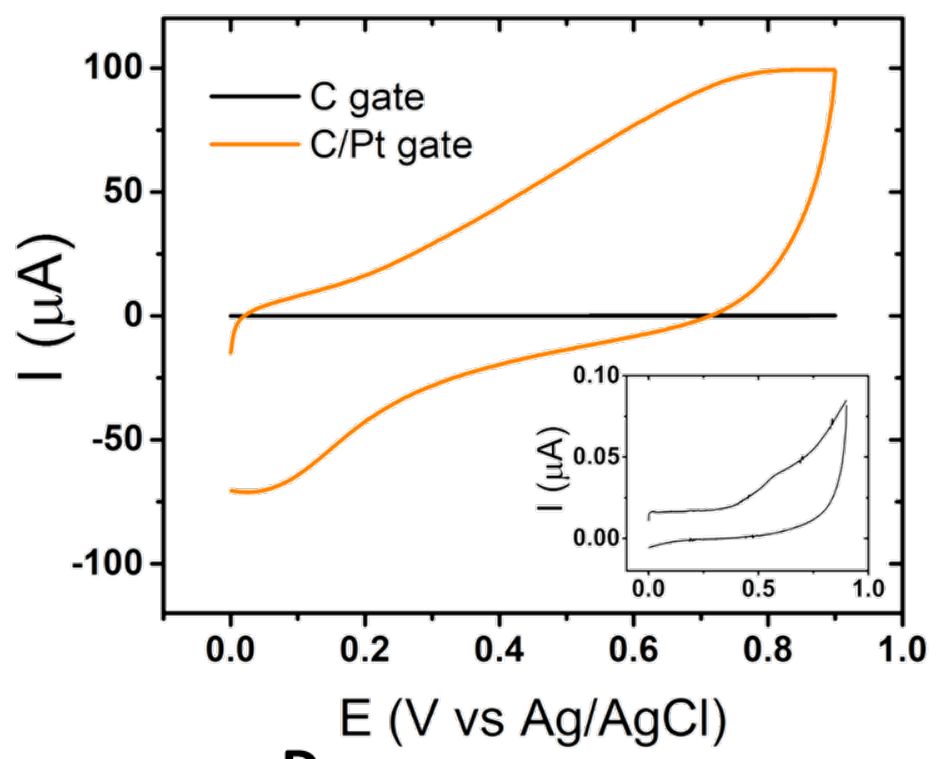

D

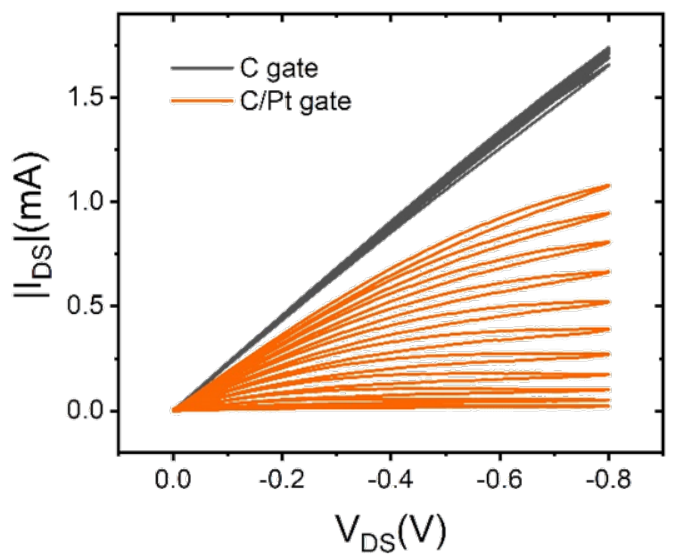

Figure S1: Platinum functionalization step. (A) Optical microscopy image of the gate presenting a platinum layer on top of the carbon printed gate. (B) Cyclic voltammetry showing the capacitive behaviour of Carbon gate ( $\mathrm{C}$ gate, black curve) and Carbon gate covered with Platinum $(\mathrm{C} / \mathrm{Pt}$ gate, orange) recorded in $50 \mathrm{mM} \mathrm{PBS}$, voltage range $0 \mathrm{~V}-0.9 \mathrm{~V}$ and scan rate $50 \mathrm{mV} / \mathrm{s}$. The inset shows a magnification of the $\mathrm{C}$ gate, black curve. $(\mathrm{C}, \mathrm{D})$ Overlay of transfer measured at fixed $\mathrm{V}_{\mathrm{SD}}=-0.4 \mathrm{~V}(\mathrm{C})$ and output characteristics measured in $50 \mathrm{mM}$ PBS buffer, 
$\mathrm{V}_{\mathrm{GS}}$ scanning from $0.0 \mathrm{~V}$ to $+1.0 \mathrm{~V}$ with steps of $+0.1 \mathrm{~V}(\mathrm{D})$ for $\mathrm{C}$ and $\mathrm{C} / \mathrm{Pt}$ gated OECT in black and orange, respectively.
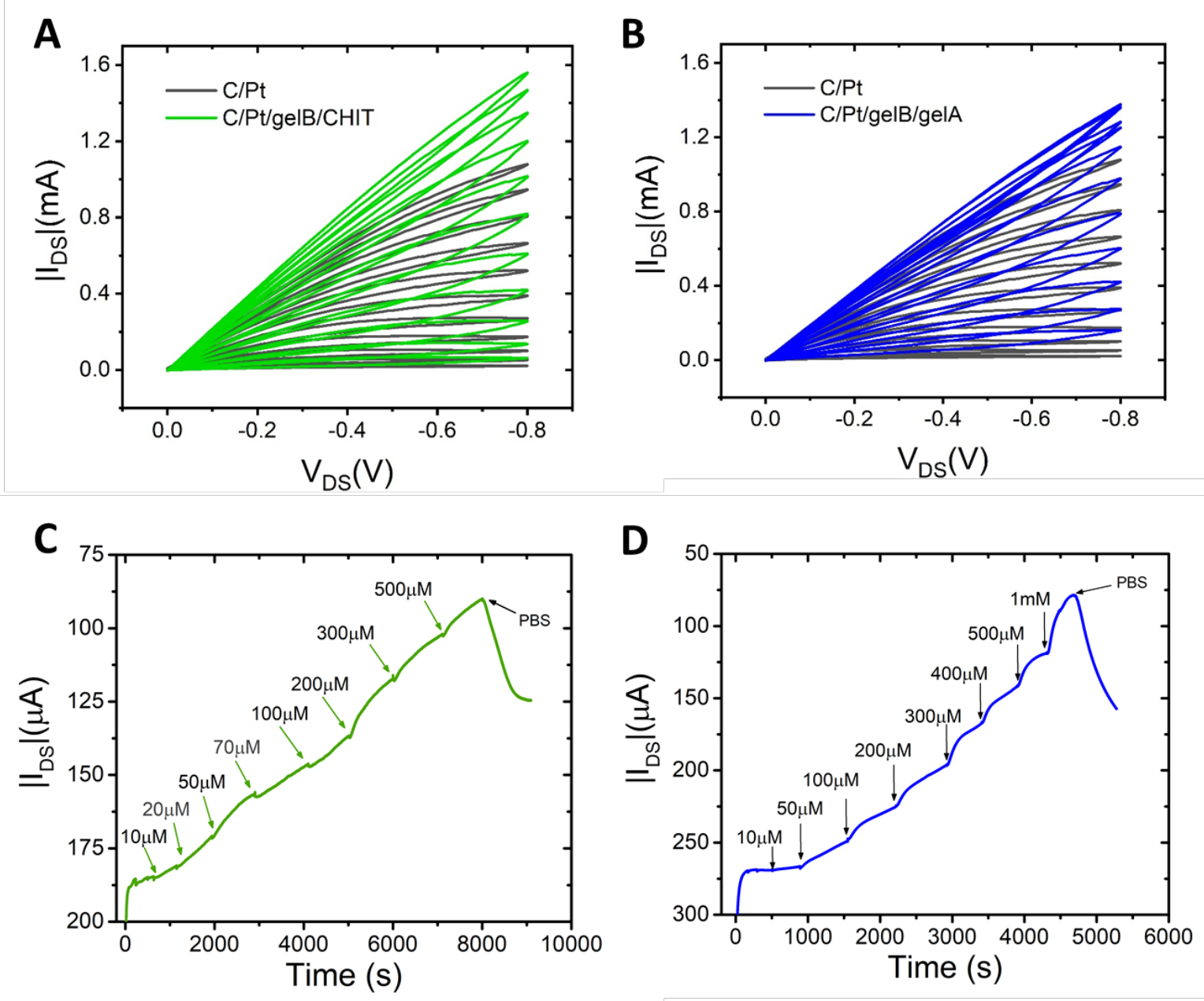

Figure S2: Chitosan and Gelatin-A performance. (A) Output characteristics of $\mathrm{C} / \mathrm{Pt} / \mathrm{GelB} / \mathrm{Chit}+\mathrm{UOx}$ and $\mathrm{C} / \mathrm{Pt} / \mathrm{GelB} / \mathrm{GelA}+\mathrm{UOx}$ OECTs (A) and (B), respectively. Measured in $50 \mathrm{mM}$ PBS buffer, $\mathrm{V}_{\mathrm{GS}}$ scanning from $0.0 \mathrm{~V}$ to $+1.0 \mathrm{~V}$ with steps of $+0.1 \mathrm{~V}$. (C) Drain current vs. time response of a $\mathrm{C} / \mathrm{Pt} / \mathrm{GelB} / \mathrm{Chit}+\mathrm{UOx} \mathrm{OECT}$ for increasing concentration of uric acid. (D) Drain current vs. time response of a $\mathrm{C} / \mathrm{Pt} / \mathrm{GelB} / \mathrm{GelA}+\mathrm{UOx}$ OECT for increasing concentration of uric acid. 


\section{WILEY-VCH}
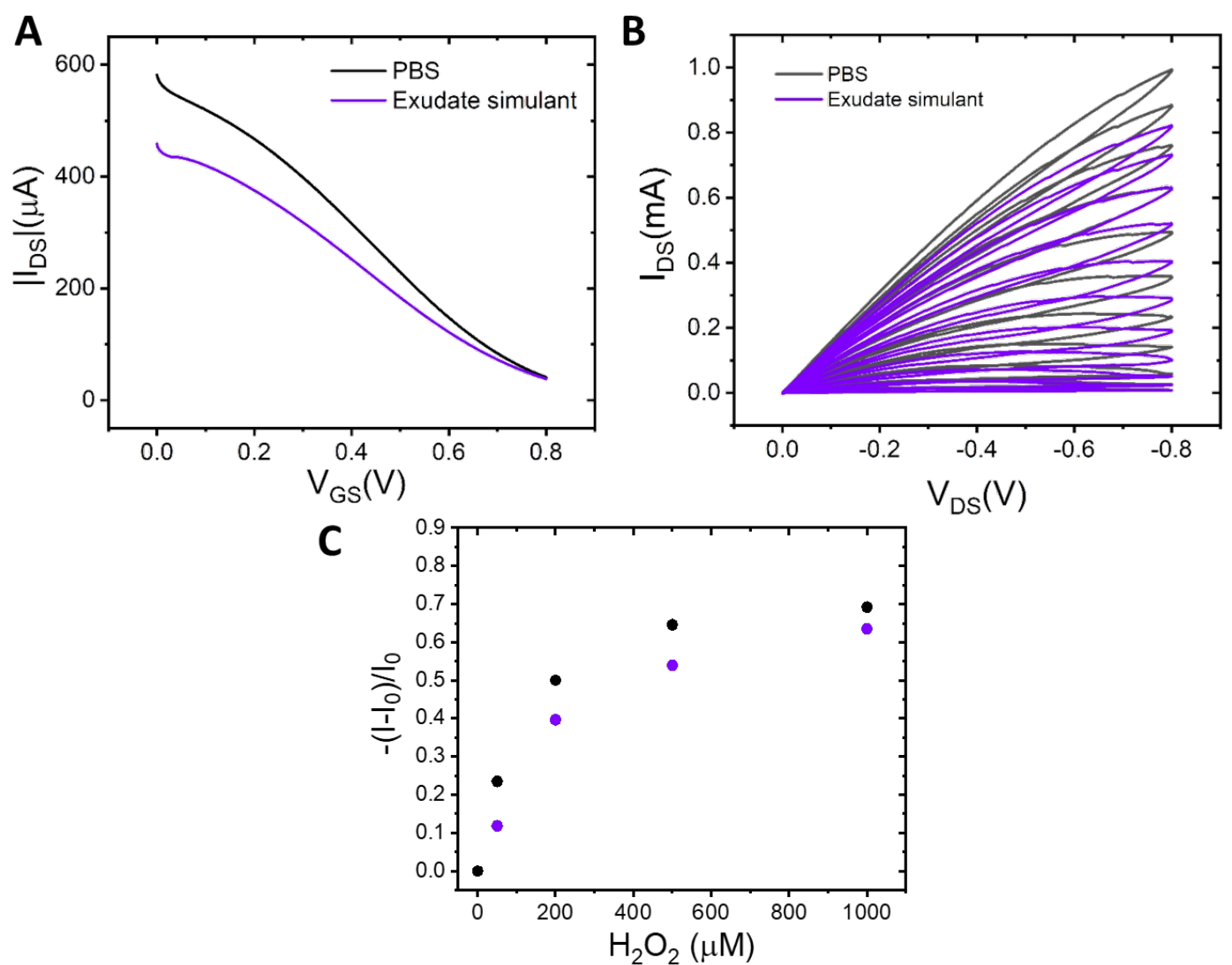

Figure S3: electrical characteristics in PBS and exudate simulant. (A) Transfer characteristics of $\mathrm{C} / \mathrm{Pt} / \mathrm{GelB} / \mathrm{GelA}+\mathrm{UOx}$ gated OECT in PBS buffer (black line) and exudate simulant (violet line) measured at fixed $\mathrm{V}_{\mathrm{SD}}=-0.4 \mathrm{~V}$. (B) Output characteristics of the functionalized OECT in PBS buffer (black line) and exudate simulant (violet line), $\mathrm{V}_{\mathrm{GS}}$ scanning from $0.0 \mathrm{~V}$ to $+1.0 \mathrm{~V}$ with steps of $+0.1 \mathrm{~V}$. (C) Normalized response of the biosensor to increasing concentrations of $\mathrm{H}_{2} \mathrm{O}_{2}$ in PBS (black dots) and exudate simulant (violet dots). 


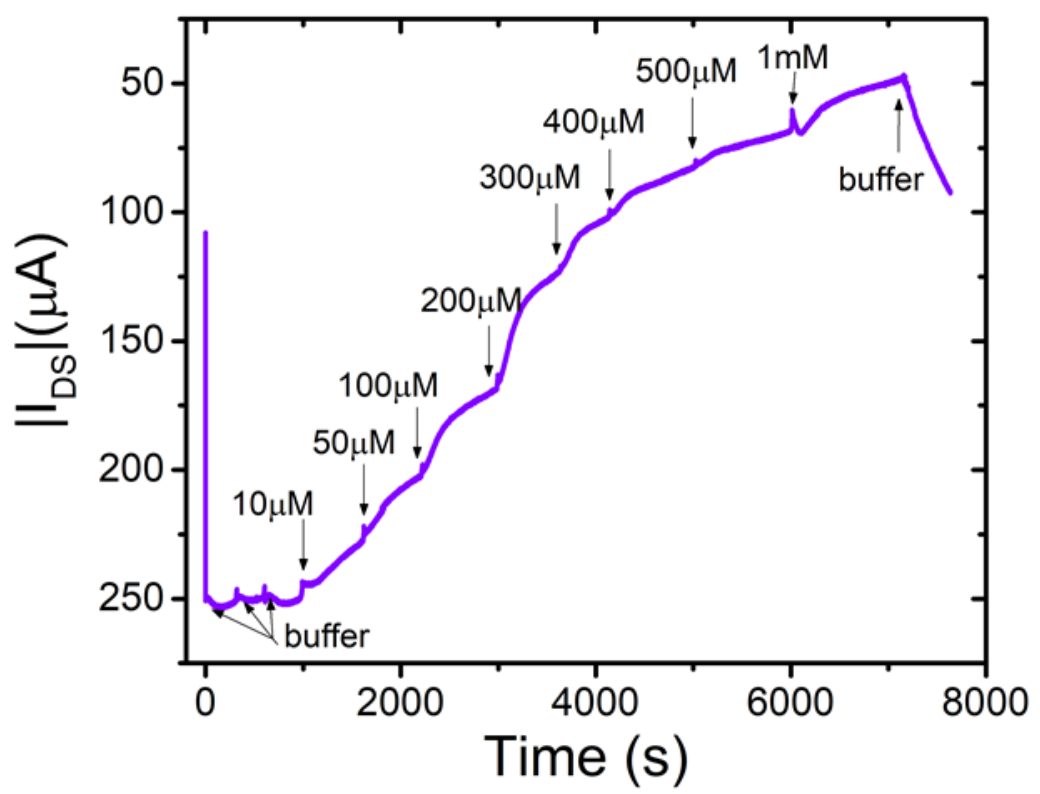

Figure S4: Uric acid detection in complex media. Drain current versus time response of a $\mathrm{C} / \mathrm{Pt} / \mathrm{GelB} / \mathrm{GelA}+\mathrm{UOx}$ gated OECT operated in exudate simulant, at different UA concentrations. 